\title{
Instrumento para Identificar Características Personales y Didáctica Utilizadas por los Docentes en la Formación de Emprendedores
}

\author{
Gloria Y. Rojas ${ }^{1}$, Vanessa Pertuz ${ }^{2}$, Astrid Navarro' y Liliana T. Quintero' \\ (1) Universidad de Santander, Valledupar, Colombia. \\ (2) Universidad de Santander, Facultad de Ingenierías, Grupo de Investigación Nuevas Tecnologías-UDES, \\ Valledupar, Colombia. (e-mail: van.pertuz@mail.udes.edu.co)
}

Recibido Jun. 14, 2018; Aceptado Ago. 21, 2018; Versión final Oct. 1, 2018, Publicado Abr. 2019

\begin{abstract}
Resumen
El objetivo del estudio fue diseñar un instrumento para identificar características personales y didácticas utilizadas por los docentes en la formación de emprendedores. El instrumento fue validado mediante una prueba piloto aplicada a 52 docentes universitarios. La validez de contenido se estableció mediante el juicio de expertos. La confiabilidad de instrumento se estimó mediante el coeficiente de Alfa de Cronbach. Con esta prueba se obtuvo una confiabilidad de 0,88 . Adicionalmente, se realizó el análisis factorial para la verificación de la dimensionalidad del instrumento propuesto y los análisis de ítems, obteniendo un resultado de la medida de adecuación muestral de 0,958 (índice Kaiser-Meyer-Olkin, KMO). De esta manera, el porcentaje de la varianza total explicada corresponde a 99,88\%. La estructura de instrumento quedó conformada por 28 ítems, mediante una escala tipo Likert. El instrumento de recolección de datos presentado en el artículo aporta una herramienta para el desarrollo de programas de formación a formadores en emprendimiento y, consecuentemente, contribuye al desarrollo de actitudes emprendedoras en estudiantes, mediante la práctica docente.
\end{abstract}

Palabras clave: competencias docentes; formador en emprendimiento; educación emprendedora; emprendimiento universitario

\section{Instrument to Identify Personal and Didactic Characteristics Used by Teachers in the Training of Entrepreneurs}

\begin{abstract}
The objective of this study was to design an instrument to identify personal and didactic characteristics used by teachers in the training of entrepreneurs. The instrument was validated through a pilot test applied to 52 faculty teachers. The content was validated through the judgment of expert professionals. The reliability of the instrument was estimated using the Cronbach's Alpha coefficient. This test reports a reliability of 0.88 . Additionally, factorial analysis was performed to verify the dimensionality of the proposed instrument and the item analyzed. The instrument obtains a result of 0.958 for the index Kaiser-Meyer-Olkin (KMO). In this way, the percentage of the total variance explained corresponds to $99.88 \%$. The instrument structure was made up by 28 items, using a Likert scale. The data collection instrument presented in this paper provides a tool for the development of programs for entrepreneurship trainers and, consequently, contributes to the development of entrepreneurial attitudes in students, through the teaching practice.
\end{abstract}

Keywords: teacher skills; trainer in entrepreneurship; entrepreneurship culture; university entrepreneurship 


\section{INTRODUCCIÓN}

En referencia al enfoque de los estudios sobre emprendimiento, en la literatura se presentan diversas perspectivas. El trabajo de Trivedi (2017) destaca que, cada vez más, la literatura sobre el emprendimiento se ha centrado en modelos basados en la intención. De otro lado, en referencia a las teorías que soportan los estudios de emprendimiento, Roy et al. (2017) y Tiwari et al. (2017) fundamentan el estudio de emprendimiento en India, en la teoría de del comportamiento planificado (TPB) propuesta por Ajzen. Igualmente, Trivedi (2017) retoma la teoría TPB para analizar el emprendimiento en los estudiantes de posgrado en gerencia de India, Singapur y Malasia. De otro lado, los autores Abbasi et al. (2017) proponen un modelo de adopción de carrera emprendedora, derivado de la teoría social cognitiva, según la cual, las condiciones de contexto conjuntamente con la personalidad emprendedora resultan fundamentales en la formación de emprendedores. De otro lado, Trivedi (2017) analiza como factores del emprendimiento: las barreras endógenas, el ambiente exógeno, el ambiente universitario y el apoyo en la intención empresarial entre los estudiantes de gestión. Otro de los factores destacados en la literatura corresponden a: el conocimiento empresarial requerido (Roy et al., 2017) y la percepción de los estudiantes frente a su experiencia de aprendizaje en la universidad (Tognazzo et al., 2017), (Tiwari et al., 2017) y (Singh et al., 2017). Por su parte, Singh et al. (2017) propone la necesidad de estudiar los factores de intención emprendedora desde una perspectiva holística, validando la influencia de los factores individuales y socioculturales en la intención emprendedora. Por su parte, Sher et al. (2017) destaca como factores de la intención emprendedora: la actitud, el apoyo familiar, la aceptación mental y el control conductual percibido; mientras que Arunkumar et al. (2018) enfatiza en la importancia de la actitud emprendedora.

Concretamente, los resultados de la revisión de literatura sobre emprendimiento universitario en Colombia, realizado por el Ministerio de Comercio, Industria y Turismo y Asociación Colombiana de Universidades (2014), indican que la función más desarrollada en las universidades es la docencia, seguida por la extensión y, finalmente la investigación. Adicionalmente, la investigación en referencia concluye que el emprendimiento ha modificado las estructuras y los currículos de las universidades, no obstante, sigue siendo un campo en desarrollo, con diferentes énfasis y especificidades. La situación anterior dificulta la implementación de estrategias para el desarrollo de una educación emprendedora, por lo que se hace necesario trabajar en el diseño de lineamientos para que, desde la Educación Superior, se contribuya a la generación de capacidades que permitan el salto de emprendimientos por necesidad y tradicionales a los de base tecnológica, dinámicos e innovadores. Adicionalmente, la educación en emprendimiento se asocia con técnicas y métodos de enseñanza específicos, que incluyen el contacto presencial de los estudiantes y la alineación del conocimiento con las necesidades del mercado laboral (Stadler y Smith, 2017). Así, la literatura destaca experiencias metodológicas orientadas a fomentar las habilidades y actitudes empresariales, como, por ejemplo, la metodología de enseñanza denominada "Aprender a ser", propuesta por los autores Daniel et al. (2017); que plantea prácticas y directrices para la creación de espacios curriculares orientados al emprendimiento. Por su parte, Munawaroh (2018) comprueba la existencia de un efecto positivo del método de aprendizaje basado en problemas sobre la capacidad de pensamiento crítico de los estudiantes en la práctica de emprendimiento. De otro lado, Verzat et al. (2017) propone pautas metodológicas para el diseño pedagógico de la educación emprendedora, a través de una mejor comprensión de los factores emocionales de los estudiantes emprendedores; mientras que David et al. (2017) orientan las pautas a los catalizadores para el desarrollo del emprendimiento. En este sentido, los estudios de Sher et al. (2017) y Papadaki et al. (2017) enfatizan en la necesidad de promover la educación emprendedora en los estudiantes universitarios, desde la labor académica.

En este orden de ideas, la educación emprendedora implica entender los importantes desafíos que ésta plantea, uno de ellos es un cambio conceptual de la noción de emprendimiento asumiendo la perspectiva integral de un conjunto de competencias válidas para todos los aspectos de la vida, tanto personal, como ciudadano o laboral. En este contexto, las nuevas condiciones de competitividad en un mundo globalizado deben permitir a los jóvenes tomar decisiones sobre su destino profesional con un mayor abanico de oportunidades. Por lo anterior, la enseñanza del emprendimiento hoy en día, se basa en la creación y aplicación de modelos teóricos y prácticos para explicar las razones por las cuales ciertas decisiones se toman y fomentar el emprendimiento como una manera de pensar y de actuar; postulado que se encuentra en concordancia con lo establecido en la Ley 1014 de 2006, direccionada al fomento de la cultura del emprendimiento en Colombia (Congreso de Colombia, 2006). De este modo, la promoción del espíritu empresarial desempeña un papel importante en la sociedad y existe una relación directa comprobada entre el espíritu empresarial y el crecimiento económico (Papadaki et al., 2017).

Desde esta perspectiva, autores como Campos y Méndez (2012), Durand (2012) Caldera et al. (2004), Del Solar (2010), Bello (2008), Orti y Clares (2007) y Cámara de Comercio de Bogotá (2009) han identificado algunas características del docente formador en emprendimiento, quien como miembro de la comunidad educativa, tiene la responsabilidad de desarrollar los tres primeros procesos de la cadena de valor del 
emprendimiento: sensibilización, identificación y formulación de proyectos acorde con lo establecido por la Ley 1014 de 2006 en Colombia (Congreso de Colombia, 2006). En este orden de ideas, surge la pregunta ¿cómo enseñar emprendimiento?, para lo cual Jaramillo (2008) propone una mirada sobre el docente, y lo define como un ciudadano de la sociedad del conocimiento que debe asumir constantemente retos ante los cambios y responder de manera positiva con nuevas ideas y formas de hacer las cosas, con iniciativa y decisión ante las distintas circunstancias; es decir, un docente emprendedor debe ser también una persona competente que sabe y sabe hacer, que hace posible un aprendizaje continuo de sus estudiantes para el desarrollo de competencias específicas.

De otro lado, los estudios previos presentan instrumentos de medición del emprendimiento desde varios enfoques, el primero de ellos, desde la perspectiva social, específicamente, Capella et al. (2016) miden el emprendimiento social en estudiantes universitarios. De la misma Portuguez et al. (2018) presentan el diseño y validación de un test de evaluación del potencial en emprendimiento social, validado en emprendedores pertenecientes a una red de incubadoras de una universidad en México. Un segundo enfoque de los instrumentos se orienta a la medición de emprendimiento en estudiantes, a manera de ejemplo, Muñiz et al. (2014) desarrollan y validan un instrumento para evaluar los rasgos fundamentales de la personalidad emprendedora de los jóvenes, por su parte, Pedrosa et al. (2016) proponen un test adaptativo informatizado para la aplicación del instrumento anteriormente referenciado. En el mismo sentido, Oliver y Galiana (2015) realizan el diseño y la validación de una Escala de Actitudes Emprendedoras para Estudiantes (EAEE), la cual evalúa los siguientes factores: proactividad, ética profesional, empatía, innovación, autonomía y asunción de riesgos. Similarmente, García (2010) examina la validez factorial del cuestionario de orientación emprendedora (COE) en estudiantes universitarios de España, Portugal, México, Brasil y Argentina; mientras que Rodríguez et al. (2018) describen los elementos y las relaciones que explican la intención emprendedora en estudiantes universitarios.

Un tercer enfoque se orienta a la medición de características específicas de los emprendedores, por ejemplo, Manzano y Ayala (2013) analizan las propiedades psicométricas de la escala de resiliencia de Connor y Davidson (CD-RISC) en una muestra de emprendedores españoles, al considerarla un factor que explica el éxito de los emprendimientos. Por su parte, la investigación de Mueller y Thomas (2001) analizan la influencia de la cultura de diferentes países en referencia al locus de control interno y la innovación, concluyendo que algunas culturas son más propicias para emprendimiento que otras. Adicionalmente, Bernal y Cárdenas (2017) enfatizan en que el éxito de la educación emprendedora depende de factores personales, además de ciertos factores externos, que conforman el potencial emprendedor de una persona. Adicionalmente, se destaca el programa de investigación Global Entrepreneurship Monitor, que realiza una evaluación integral del rol del emprendimiento en el crecimiento económico nacional (Reynolds et al., 2005).

En suma, la revisión de literatura destaca que la medición de emprendimiento se concentra en los estudiantes, el enfoque social y las características específicas que favorecen el emprendimiento. Pocos estudios consideran la medición del emprendimiento desde la perspectiva del docente. Al respecto, Bernal y Cárdenas (2017) destacan que la perspectiva del docente en la educación emprendedora no ha sido suficientemente atendida, pese al rol crucial de los mismos en la implementación de este tipo de educación. El presente artículo aborda la medición del emprendimiento desde la perspectiva del docente, mediante un instrumento que permite, a las instituciones de Educación Superior, identificar características personales y didácticas utilizadas por los docentes en su práctica pedagógica para lograr el fomento de la cultura del emprendimiento en los estudiantes, propendiendo por mejorar la manera de preparar emprendedores universitarios.

\section{COMPETENCIAS Y EMPRENDIMIENTO}

Las competencias del docente son esenciales en la enseñanza del emprendimiento, así como el significado que tiene "formar emprendedores". Al respecto, la literatura de emprendimiento específicamente orientada a la formación de formadores, es incipiente y se encuentra en una etapa de construcción teórica. Adicionalmente, a partir de una revisión sistemática, los autores Huang et al. (2018) destacan que, a pesar de la amplia adopción del espíritu empresarial por parte de los programas de ingeniería de los Estados Unidos, ha habido pocos avances en la forma de medir las influencias de la educación empresarial en los estudiantes de ingeniería. En este sentido, Rodríguez et al. (2018) señalan que las instituciones de educación superior deben dirigir los esfuerzos para mejorar las habilidades empresariales de los estudiantes. De la misma manera, el estudio de Sepúlveda et al. (2017) compara las características emprendedoras personales y la alfabetización económica en estudiantes de dos universidades en Chile, destacando la necesidad de "revisar los planes de estudios de carreras universitarias cuyo objetivo explícito es la formación de profesionales emprendedores, de tal manera que incorporen de manera efectiva contenidos y competencias vinculadas al emprendimiento y a la alfabetización económica" p.107. 


\section{Competencias}

Para la construcción del instrumento propuesto en el presente artículo, orientado a la identificación de características personales y didáctica utilizada por los docentes en la formación de emprendedores, una competencia se define como saber hacer en contexto. Frente al enfoque de competencias, Agudo et al. (2013), señalan que "las competencias facilitan el desarrollo de una verdadera educación integral al englobar todas las dimensiones del ser humano (saber, saber hacer y saber ser y estar), y suponen un referente para la superación de una enseñanza meramente academicista” p.40.

En este caso en particular, el contexto donde se pone en evidencia la formación y desarrollo de las competencias, es el proceso pedagógico realizado por un docente para desarrollar actitudes y habilidades emprendedoras con los estudiantes universitarios. Si bien existen diferentes aproximaciones y enfoques al concepto de competencia, hay unos elementos que son comunes en varias propuestas y autores. Estos elementos se han venido consolidando como los componentes fundamentales de las competencias y son el saber (conocimientos), el hacer (habilidades) y el ser (actitudes y valores). Para una mejor comprensión de los componentes de las competencias, es decir de los atributos que causan desempeños superiores, se adopta el modelo del lceberg propuesto por Spencer y Spencer (1993), el cual indica que los conocimientos y las habilidades se encuentran en la parte superior, en la superficie; mientras que en la parte más profunda del iceberg se ubican el rol social, la imagen de sí mismo, los rasgos y motivos (Figura 1). Este enfoque permite un abordaje práctico desde la formación y evaluación de las competencias del docente formador en emprendimiento (Alles, 2006).

Para efectos del presente artículo se adapta la definición de las competencias propuesta por Spencer y Spencer (1993), como sigue: habilidades, capacidad de un docente para aplicar las técnicas y métodos para la enseñanza del emprendimiento (desde el enfoque constructivista: aprendizaje basado en proyectos, aprendizaje basado en problemas y aprendizaje basado en casos); conocimientos, la información que tiene el docente en temas relacionados con emprendimiento; rol social, el patrón de comportamiento del docente que es reforzado por su grupo de referencia; imagen de sí mismo, concepto que el docente tiene de sí mismo en función de su identidad, personalidad y valor; rasgos, aspecto típico del comportamiento del docente; motivos, lo que dirige el comportamiento del docente en el fomento de la cultura de emprendimiento.

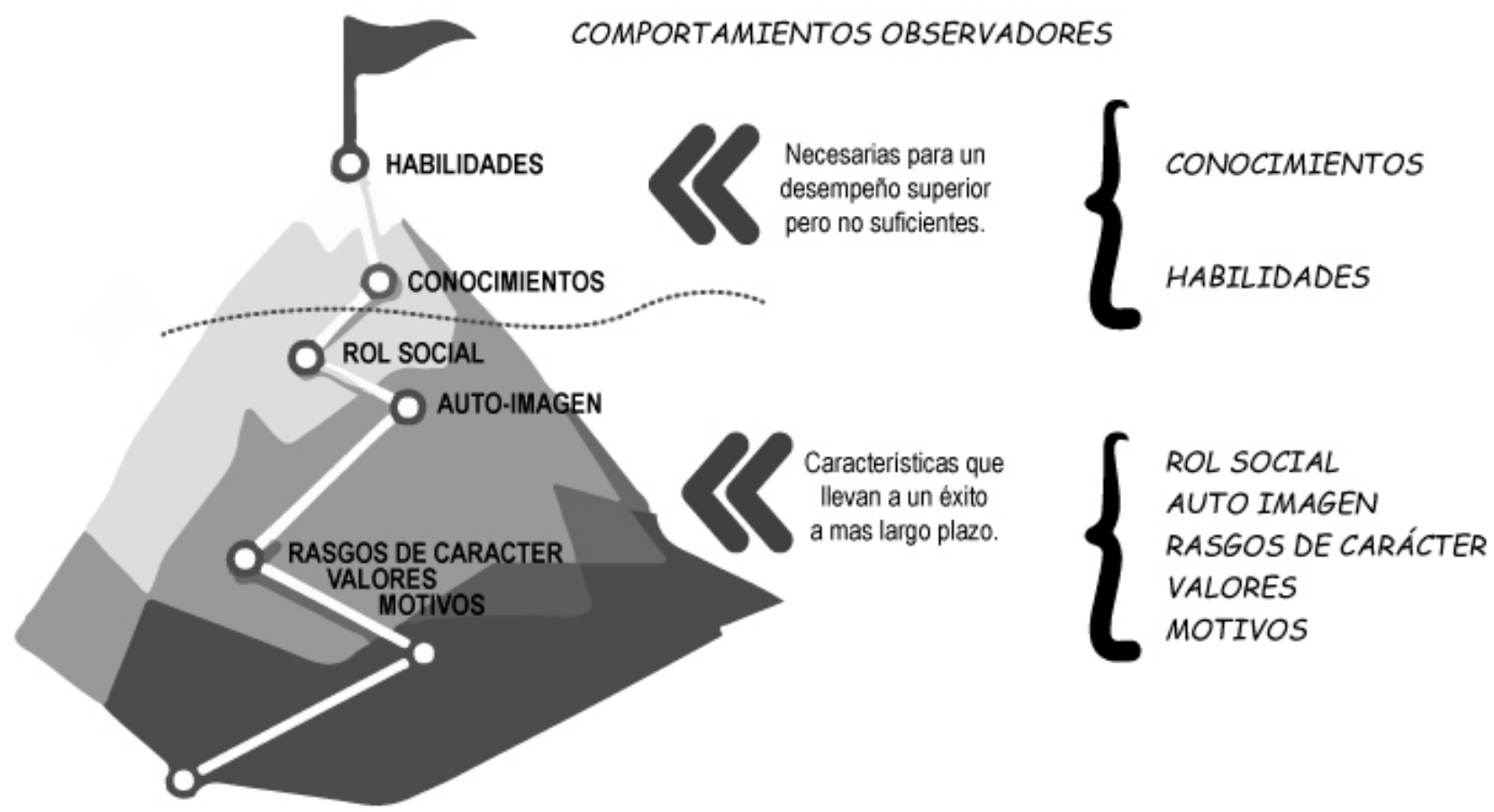

Fig. 1: Modelo Iceberg de definición de competencias propuesto por Spencer y Spencer (1993), tomado de Alles (2006).

Desde este enfoque, se deben considerar los componentes de las competencias dentro del proceso de aprendizaje, con el objeto de diseñar estrategias didácticas que posibiliten experiencias significativas, teniendo en cuenta los elementos cognoscitivos, afectivos y psicomotrices de los estudiantes universitarios para formar el espíritu emprendedor. 


\section{METODOLOGIA}

Para la construcción del instrumento propuesto en el presente artículo se consideran las siguientes fases: Como primera fase, se realiza la revisión de literatura orientada a la identificación de las características del docente que forma emprendedores. Posteriormente, se definen cada uno de las características de acuerdo con los autores considerados en la literatura y se identifica a que componente del modelo de competencias de Spencer y Spencer (Figura 1) corresponden cada una de las características identificadas. Como segunda fase, se plantearon cada una de los ítems que permiten medir las diferentes características del docente que forma emprendedores en el marco del modelo de competencias considerado como referente. Como tercera fase, se realizó el cálculo de la validez y la confiabilidad del instrumento propuesto, tomando como referente metodológico el trabajo de Baeza et al. (2016). Como cuarta fase, se realizó el análisis factorial para la verificación de la dimensionalidad del instrumento propuesto. Como quinta fase, se presentan los pasos para facilitar la aplicación del instrumento de recolección de datos propuesto en el presente artículo.

\section{RESULTADOS Y DISCUSIÓN}

De acuerdo con la primera fase de la metodología, se realizó una revisión de literatura orientada a la identificación de las características que tienen los docentes que forman emprendedores. Así, se consideran como representativos los aportes de Campos y Méndez (2012), Durand (2012), Caldera et al. (2004), Del Solar (2010), Bello (2008), Orti y Clares (2007) y Cámara de Comercio de Bogotá (2009). Posteriormente, se analizaron los componentes de las competencias definidas por Spencer y Spencer (1993) (Figura 1) desde el contexto de la investigación, para relacionarlos con las características identificadas por los autores (Tabla 1).

Tabla 1: Relación de las características del docente que forma emprendedores y los componentes de las competencias

\begin{tabular}{|c|c|c|c|c|}
\hline$N^{\circ}$ & Característica & Definición de la característica & Autores & $\begin{array}{l}\text { Componente } \\
\text { de la } \\
\text { competencia } \\
\text { (Spencer y } \\
\text { Spencer) }\end{array}$ \\
\hline 1 & Conocimiento & $\begin{array}{l}\text { Genera las condiciones para el desarrollo del } \\
\text { aprendizaje significativo en los estudiantes } \\
\text { mediante la trasmisión de orientaciones, medios, } \\
\text { experiencias, estrategias y recursos pedagógicos } \\
\text { desde un proceso reflexivo de construcción del } \\
\text { conocimiento }\end{array}$ & $\begin{array}{c}\text { Del Solar (2010), Durand } \\
\text { (2012), Cámara de Comercio } \\
\text { de Bogotá (2009), Caldera et } \\
\text { al. (2004) }\end{array}$ & Conocimientos \\
\hline 2 & Flexibilidad & $\begin{array}{l}\text { Introduce modificaciones y ajustes a la } \\
\text { planificación en función de la evolución del } \\
\text { proceso de enseñanza, integrando los temas y } \\
\text { prácticas de interés por parte de los estudiantes. }\end{array}$ & $\begin{array}{l}\text { Del Solar (2010), Durand } \\
\text { (2012), Cámara de Comercio } \\
\text { de Bogotá (2009), Jaramillo } \\
\text { (2008), Caldera et al. (2004) }\end{array}$ & $\begin{array}{l}\text { Rasgos de } \\
\text { carácter }\end{array}$ \\
\hline 3 & Tolerancia & $\begin{array}{l}\text { Promueve dentro del proceso de enseñanza- } \\
\text { aprendizaje el respeto hacia las ideas o prácticas } \\
\text { diferentes o contrarias a las propias }\end{array}$ & $\begin{array}{l}\text { Del Solar (2010), Durand } \\
\text { (2012), Cámara de Comercio } \\
\text { de Bogotá (2009), Orti y } \\
\text { Clares (2007), Bello (2008) }\end{array}$ & Valores \\
\hline 4 & Respeto & $\begin{array}{l}\text { Promueve espacios de discusión y análisis } \\
\text { fundamentados en el reconocimiento y la } \\
\text { importancia de las ideas de los involucrados en el } \\
\text { proceso de enseñanza aprendizaje. }\end{array}$ & $\begin{array}{l}\text { Del Solar (2010), Durand } \\
\text { (2012), Cámara de Comercio } \\
\text { de Bogotá (2009), Orti y } \\
\text { Clares (2007), Bello (2008) }\end{array}$ & Valores \\
\hline 5 & Responsabilidad & $\begin{array}{l}\text { Promueve dentro del proceso de enseñanza- } \\
\text { aprendizaje que los estudiantes asumen las } \\
\text { consecuencias de sus actos. }\end{array}$ & $\begin{array}{l}\text { Del Solar (2010), Durand } \\
\text { (2012), Cámara de Comercio } \\
\text { de Bogotá (2009), Orti y } \\
\text { Clares (2007), Bello (2008) }\end{array}$ & Valores \\
\hline 6 & $\begin{array}{l}\text { La enseñanza se } \\
\text { centra en el } \\
\text { alumno }\end{array}$ & $\begin{array}{l}\text { Orienta al alumno hacia la autonomía y la auto- } \\
\text { dirección del proceso construcción de } \\
\text { conocimiento, proporcionado las bases requeridas } \\
\text { y favoreciendo los espacios de crítica, trabajo en } \\
\text { equipo y auto formación. }\end{array}$ & $\begin{array}{l}\text { Del Solar (2010), Durand } \\
\text { (2012), Cámara de Comercio } \\
\text { de Bogotá (2009), Jaramillo } \\
\text { (2008), Caldera et al. (2004) }\end{array}$ & Habilidades \\
\hline 7 & Facilitador & $\begin{array}{l}\text { Contribuye a que el estudiante desarrolle sus } \\
\text { competencias mediante la motivación, la } \\
\text { orientación y herramientas de ayuda para el } \\
\text { desarrollo efectivo del proceso de aprendizaje }\end{array}$ & $\begin{array}{c}\text { Durand (2012), Cámara de } \\
\text { Comercio de Bogotá (2009), } \\
\text { Jaramillo (2008), Campos y } \\
\text { Méndez (2012). }\end{array}$ & Habilidades \\
\hline 8 & $\begin{array}{l}\text { Identifican } \\
\text { adecuadamente } \\
\text { las necesidades } \\
\text { de los } \\
\text { estudiantes }\end{array}$ & $\begin{array}{l}\text { Capacidad de desarrollar el proceso de } \\
\text { enseñanza en concordancia con los aspectos } \\
\text { requeridos y las expectativas de los estudiantes }\end{array}$ & $\begin{array}{c}\text { Durand (2012), Cámara de } \\
\text { Comercio de Bogotá (2009), } \\
\text { Bello (2008), Campos y } \\
\text { Méndez (2012), Caldera et al. } \\
\text { (2004) }\end{array}$ & Habilidades \\
\hline
\end{tabular}




\begin{tabular}{|c|c|c|c|c|}
\hline \multicolumn{5}{|c|}{ Tabla 1 (continuación) } \\
\hline$N^{\circ}$ & Característica & Definición de la característica & Autores & $\begin{array}{l}\text { Componente } \\
\text { de la } \\
\text { competencia } \\
\text { (Spencer y } \\
\text { Spencer) }\end{array}$ \\
\hline 9 & Problematizador & $\begin{array}{l}\text { Genera preguntas o situaciones que estimulan la } \\
\text { capacidad de análisis, indignación y reflexión de } \\
\text { los estudiantes como mecanismo para la } \\
\text { construcción del conocimiento }\end{array}$ & $\begin{array}{l}\text { Orti y Clares (2007), Bello } \\
\text { (2008), Campos y Méndez } \\
\text { (2012), Caldera et al. ( 2004) }\end{array}$ & Habilidades \\
\hline 10 & $\begin{array}{l}\text { Promotor del } \\
\text { trabajo en grupo }\end{array}$ & $\begin{array}{l}\text { Asume el grupo como el contexto idóneo para el } \\
\text { desarrollo de las habilidades de los estudiantes, } \\
\text { por lo que favorece la solidaridad, la tolerancia, la } \\
\text { interacción entre los estudiantes, la motivación, el } \\
\text { sentido de responsabilidad, la discusión y el } \\
\text { análisis. }\end{array}$ & $\begin{array}{l}\text { Del Solar (2010), Durand } \\
\text { (2012), Bello (2008) }\end{array}$ & Habilidades \\
\hline 11 & $\begin{array}{l}\text { Planifican el } \\
\text { proceso de } \\
\text { aprendizaje }\end{array}$ & $\begin{array}{l}\text { Establece anticipadamente los objetivos del } \\
\text { proceso de enseñanza, definiendo los contenidos, } \\
\text { las metodologías, las estrategias necesarias y los } \\
\text { criterios de evaluación para el dominio de las } \\
\text { competencias por parte de los estudiantes }\end{array}$ & $\begin{array}{l}\text { Del Solar (2010), Bello (2008), } \\
\text { Campos y Méndez (2012), } \\
\text { Cámara de Comercio de } \\
\text { Bogotá (2009) }\end{array}$ & Habilidades \\
\hline 12 & $\begin{array}{c}\text { Capacidad de } \\
\text { enseñar en } \\
\text { procesos y } \\
\text { contextos } \\
\text { prácticos (reales) } \\
\text { / (Ausencia de } \\
\text { clases } \\
\text { magistrales) }\end{array}$ & $\begin{array}{l}\text { Fundamenta los procesos de enseñanza- } \\
\text { aprendizaje en las experiencias previas y en la } \\
\text { generación de situaciones que permitan al } \\
\text { estudiante la construcción del conocimiento } \\
\text { mediante la interacción con el contexto practico. }\end{array}$ & $\begin{array}{l}\text { Del Solar (2010), Durand } \\
\text { (2012), Jaramillo (2008) }\end{array}$ & Habilidades \\
\hline 13 & Innovador & $\begin{array}{l}\text { Genera cambios en el desarrollo de los procesos } \\
\text { de enseñanza-aprendizaje, propiciando en los } \\
\text { estudiantes una nueva forma de observar el } \\
\text { entorno y las oportunidades que este ofrece }\end{array}$ & $\begin{array}{l}\text { Durand (2012), Cámara de } \\
\text { Comercio de Bogotá (2009), } \\
\text { Bello (2008) }\end{array}$ & Motivos \\
\hline 14 & Comunicación & $\begin{array}{l}\text { Capacidad para transmitir efectivamente las ideas } \\
\text { a los estudiantes. }\end{array}$ & $\begin{array}{l}\text { Cámara de Comercio de } \\
\text { Bogotá (2009), Bello (2008) }\end{array}$ & Habilidades \\
\hline 15 & Empatía & $\begin{array}{l}\text { Capacidad de ponerse en el lugar de los } \\
\text { estudiantes, comprendiendo sus necesidades y } \\
\text { emociones y asumiendo una actitud abierta al } \\
\text { diálogo y al cambio logrando una interacción } \\
\text { positiva con los estudiante }\end{array}$ & $\begin{array}{l}\text { Durand (2012), Orti y Clares } \\
\text { (2007) }\end{array}$ & $\begin{array}{l}\text { Rasgos de } \\
\text { carácter }\end{array}$ \\
\hline 16 & $\begin{array}{l}\text { Evalúan y } \\
\text { controlan los } \\
\text { procesos y los } \\
\text { resultados }\end{array}$ & $\begin{array}{l}\text { Incluye dentro de la metodología de proceso de } \\
\text { enseñanza-aprendizaje, la realización de } \\
\text { acciones de verificación de los objetivos } \\
\text { planteados con el objeto de garantizar la calidad } \\
\text { de proceso y tomar las decisiones de mejora } \\
\text { necesarias. }\end{array}$ & Durand (2012), Bello (2008) & Habilidades \\
\hline 17 & Motivador & $\begin{array}{l}\text { Impulsa a los estudiantes para llevar a cabo sus } \\
\text { iniciativas. }\end{array}$ & $\begin{array}{l}\text { Jaramillo (2008), Cámara de } \\
\text { Comercio de Bogotá (2009) }\end{array}$ & Motivos \\
\hline 18 & Adaptabilidad & $\begin{array}{l}\text { Se muestra receptivo a enfrentar situaciones o } \\
\text { realizar actividades diferentes a las planificadas } \\
\text { dentro del proceso de enseñanza }\end{array}$ & Bello (2008) & $\begin{array}{l}\text { Rasgos de } \\
\text { carácter }\end{array}$ \\
\hline 19 & $\begin{array}{l}\text { Trabajan bajo } \\
\text { enfoques } \\
\text { sistemáticos y } \\
\text { sustentables }\end{array}$ & $\begin{array}{l}\text { Desarrollan el proceso de enseñanza desde } \\
\text { enfoques sistemáticos y sustentables } \\
\text { relacionados con la comunidad local }\end{array}$ & Durand (2012) & Habilidades \\
\hline 20 & $\begin{array}{l}\text { Capacidad de } \\
\text { aprender }\end{array}$ & $\begin{array}{l}\text { Realiza permanente procesos de cualificación y } \\
\text { actualización dentro de su disciplina para obtener } \\
\text { un resultado efectivo del proceso enseñanza- } \\
\text { aprendizaje }\end{array}$ & $\begin{array}{l}\text { Cámara de Comercio de } \\
\text { Bogotá (2009) }\end{array}$ & $\begin{array}{l}\text { Rasgos de } \\
\text { carácter }\end{array}$ \\
\hline 21 & Promotor & $\begin{array}{l}\text { Incentiva el desarrollo de trabajos y proyectos } \\
\text { donde el estudiante aplica y pone a prueba sus } \\
\text { conocimientos y habilidades. }\end{array}$ & $\begin{array}{l}\text { Cámara de Comercio de } \\
\text { Bogotá, (2009) }\end{array}$ & Rol social \\
\hline 22 & $\begin{array}{l}\text { Capacidad de } \\
\text { trabajar en } \\
\text { equipo }\end{array}$ & $\begin{array}{l}\text { Establece relaciones de cooperación con su } \\
\text { entorno con el objeto de lograr metas comunes de } \\
\text { manera efectiva. }\end{array}$ & $\begin{array}{l}\text { Orti y Clares (2007), Caldera } \\
\text { et al. (2004), Durand (2012), } \\
\text { Campos y Méndez (2012) }\end{array}$ & $\begin{array}{l}\text { Rasgos de } \\
\text { carácter }\end{array}$ \\
\hline
\end{tabular}

Posteriormente, de acuerdo con la segunda fase de la metodología, se establecen los ítems del instrumento de recolección de datos propuesto en el presente artículo (Tabla 2). 
Tabla 2: Estructura del instrumento para identificar características personales y didáctica utilizada por los docentes en la formación de emprendedores, a partir de los componentes de las competencias

\begin{tabular}{|c|c|c|}
\hline $\begin{array}{l}\text { Componente } \\
\text { de la } \\
\text { competencia } \\
\text { (Spencer y } \\
\text { Spencer) }\end{array}$ & $\begin{array}{l}\text { Características del } \\
\text { docente que forma } \\
\text { emprendedores }\end{array}$ & Ítems \\
\hline \multirow{2}{*}{ Conocimiento } & \multirow{2}{*}{ 1. Experticia } & 1. Utilizo ejemplos relacionando el tema de clase con el campo profesional \\
\hline & & 2. Reconozco y comprendo quién es y qué hace un emprendedor \\
\hline \multirow{14}{*}{ Habilidades } & \multirow{2}{*}{$\begin{array}{l}\text { 1. La enseñanza se } \\
\text { centra en el alumno }\end{array}$} & $\begin{array}{l}\text { 3. Mis estudiantes realizan actividades o desarrollan casos, seleccionados } \\
\text { por ellos mismos }\end{array}$ \\
\hline & & 4. Promuevo procesos de auto evaluación y co-evaluación \\
\hline & $\begin{array}{l}\text { 2. Promotor del trabajo } \\
\text { en grupo }\end{array}$ & $\begin{array}{l}\text { 5. Posibilito la participación de todos los alumnos en todas las actividades y } \\
\text { tareas, estableciendo las bases para que el desarrollo del proyecto se base } \\
\text { en estrategias de cooperación }\end{array}$ \\
\hline & 3. Facilitador & $\begin{array}{l}\text { 6. Colaboro y guío a los estudiantes entregándole herramientas y medios } \\
\text { que faciliten su aprendizaje }\end{array}$ \\
\hline & \multirow[t]{2}{*}{ 4. Problematizador } & $\begin{array}{l}\text { 7. Promuevo en el estudiante la búsqueda de diversas alternativas para la } \\
\text { solución de un problema dado }\end{array}$ \\
\hline & & 8. Promuevo la competencia entre mis estudiantes \\
\hline & $\begin{array}{l}5 . \text { Identifica } \\
\text { adecuadamente las } \\
\text { necesidades de los } \\
\text { estudiantes }\end{array}$ & $\begin{array}{l}\text { 9. Identifico las expectativas del estudiante, tanto desde la perspectiva } \\
\text { académica como desde la perspectiva del emprendimiento }\end{array}$ \\
\hline & $\begin{array}{l}\text { 6. Trabajan bajo } \\
\text { enfoques sistemáticos } \\
\text { y sustentables }\end{array}$ & $\begin{array}{l}\text { 10. Trabajo con enfoques sistemáticos y sustentables relacionados con la } \\
\text { comunidad }\end{array}$ \\
\hline & $\begin{array}{l}\text { 7. Planificar el proceso } \\
\text { de aprendizaje }\end{array}$ & $\begin{array}{l}\text { 11. Planifico mi actividad educativa de forma coordinada con el resto del } \\
\text { profesorado }\end{array}$ \\
\hline & $\begin{array}{l}\text { 8. Evalúan y controlan } \\
\text { los procesos y los } \\
\text { resultados }\end{array}$ & $\begin{array}{l}\text { 12. Mi labor docente se orienta a obtener resultados y conseguir objetivos y } \\
\text { metas }\end{array}$ \\
\hline & \multirow{2}{*}{$\begin{array}{c}\text { 9. Capacidad de } \\
\text { enseñar en procesos y } \\
\text { contextos prácticos/ } \\
\text { (Ausencia de clase } \\
\text { magistral) }\end{array}$} & $\begin{array}{l}\text { 13. Mis estudiantes explican cómo se relaciona lo que aprende en la } \\
\text { Universidad con el mundo real }\end{array}$ \\
\hline & & $\begin{array}{l}\text { 14. Genero espacios de práctica donde los estudiantes tengan la } \\
\text { oportunidad de aprender en contextos reales }\end{array}$ \\
\hline & $\begin{array}{l}\text { 10. Capacidad de } \\
\text { trabajar en equipo }\end{array}$ & $\begin{array}{l}\text { 15. Establezco relaciones de cooperación y preocupación al trabajar con } \\
\text { otros para conseguir metas comunes }\end{array}$ \\
\hline & 11. Comunicación & $\begin{array}{l}\text { 16. Escucho con atención } \\
\text { Se transmitir mis ideas }\end{array}$ \\
\hline Rol social & 1. Promotor & $\begin{array}{l}\text { 17. Incentivo el desarrollo de trabajos y proyectos donde el estudiante aplica } \\
\text { y pone a prueba su intención emprendedora }\end{array}$ \\
\hline \multirow{5}{*}{$\begin{array}{l}\text { Rasgos de } \\
\text { carácter }\end{array}$} & \multirow{2}{*}{ 1. Flexibilidad } & $\begin{array}{l}\text { 18. En la clase brindo espacios que permitan integrar temas y prácticas de } \\
\text { interés por parte de los estudiantes, relacionados a la materia }\end{array}$ \\
\hline & & $\begin{array}{l}\text { 19.confronto las opiniones de los alumnos con las mías buscando establecer } \\
\text { un acuerdo intermedio }\end{array}$ \\
\hline & 2. Adaptabilidad & $\begin{array}{l}\text { 20. Me muestro abierto a enfrentar situaciones distintas o a las que no estoy } \\
\text { acostumbrado dentro de la rutina de trabajo }\end{array}$ \\
\hline & 3. Empatía & $\begin{array}{l}21 \text { Tengo la capacidad de ponerme en el lugar de los alumnos y comprender } \\
\text { sus motivos }\end{array}$ \\
\hline & $\begin{array}{l}\text { 4. Capacidad de } \\
\text { aprender }\end{array}$ & 22.Indago, busco información, tengo inquietud permanente por conocer más \\
\hline \multirow{3}{*}{ Valores } & 1. Tolerancia & 23. Mis estudiantes corrigen o revisan el trabajo de otro compañero \\
\hline & 2. Respeto & 24. Mis estudiantes discuten entre sí \\
\hline & 3. Responsabilidad & 25. Promuevo que los estudiantes asuman las consecuencias de sus actos \\
\hline \multirow{3}{*}{ Motivos } & \multirow{2}{*}{ 1. Innovador } & $\begin{array}{l}\text { 26. Propongo y encuentro formas nuevas y eficaces de facilitar el } \\
\text { aprendizaje de los estudiantes }\end{array}$ \\
\hline & & $\begin{array}{l}\text { 27. Oriento en mis estudiantes una nueva forma de observar el entorno y las } \\
\text { oportunidades que ofrece }\end{array}$ \\
\hline & 2. Motivador & 28. Impulso a mis estudiantes para llevar a cabo sus iniciativas \\
\hline
\end{tabular}


En la tabla 2 se presentan cada uno de los ítems que miden las características de los docentes que forman emprendedores identificadas en la literatura, agrupadas de acuerdo con los componentes de las competencias del modelo de Spencer y Spencer (Figura 1). El instrumento propuesto se dividió en seis (6) secciones según el modelo del Iceberg que exploran: Conocimiento, habilidades, rol social, rasgos de carácter, valores y motivos, que consta de 28 ítems en una escala de tipo Likert. 11 ítems del instrumento se adoptaron de Domínguez et al. (2010) y los 17 restantes son de elaboración propia (Tabla 2). De otro lado, en la aplicación del instrumento, el docente debe indicar su posición respecto a los enunciados, para lo cual se emplea una escala de Likert, donde los valores van de 0 a 4, siendo $0=$ Nunca, $1=$ Casi nunca, $2=A$ veces, 3 =Casi siempre, $4=$ Siempre.

Como tercera fase se presentan los resultados de la validez y la confiabilidad del instrumento propuesto. La validez del instrumento fue estimada mediante el juicio de expertos, y la confiabilidad del mismo, mediante el coeficiente de Alfa de Cronbach, de acuerdo con el enfoque metodológico de los trabajos de Baeza et al. (2016), Agudo et al. (2013) y Capella et al. (2016). Para establecer la validez, el instrumento se sometió al procedimiento de validez de contenido (Hernandez et al., 2014), considerando la consulta de jueces expertos, especialistas en el área de metodología, emprendimiento y pedagogía. La población considerada para efectos de la presente investigación corresponde a 1195 docentes de las tres principales universidades ubicadas en Valledupar, Colombia. Mediante muestreo aleatorio simple se estimó una muestra de 209 docentes universitarios. La confiabilidad del instrumento se midió a partir de una prueba piloto, aplicada al $25 \%$ de la muestra seleccionada. Una vez calculada la validez y la confiabilidad, se aplicó el instrumento de recolección de datos a la totalidad de la muestra, obteniendo los resultados presentados por Pertuz et al. (2016).

A partir de la prueba piloto, se obtuvo una confiabilidad de 0,88 , usando el coeficiente Alfa de Cronbach, lo cual indica que el instrumento es altamente confiable, con un alto nivel de consistencia interna. Al respecto, la confiabilidad del instrumento propuesto por Capella et al. (2016) para medir emprendimiento social en estudiantes universitarios de educación física se estimó mediante el coeficiente de Alfa de Cronbach, obteniendo un resultado de 0,809 . De otro lado, el resultado del coeficiente de alfa de Cronbach para el instrumento de emprendimiento social propuesto por Portuguez et al. (2018) corresponde a 0.873, para toda la muestra. Similarmente, el índice Alfa de Cronbach fue utilizado para determinar la confiabilidad de cada uno de los nueve factores que mide el Test de Características Emprendedoras Personales (PECs, por sus siglas en inglés) (Sepúlveda et al., 2017), asimismo, para determinar la consistencia interna de la Escala de Actitudes Emprendedoras para Estudiantes (EAEE) (Oliver y Galiana, 2015), de la escala de motivación empresarial, validada en estudiantes de ingeniería (Yi y Duval, 2018) y del cuestionario de orientación emprendedora (COE) propuesto por García (2010), obteniendo una alta fiabilidad, por encima del mínimo establecido $(0,70)$.

Como cuarta fase, se realizó el análisis factorial para la verificación de la dimensionalidad del instrumento propuesto el presente artículo y los análisis de ítems (Barbero et al., 2011). En este sentido, en la tabla 3 se prestan los resultados de análisis factorial.

Tabla 3: Análisis factorial: Prueba de KMO y Bartlett.

\begin{tabular}{|c|c|c|}
\hline \multicolumn{2}{|c|}{ Medida Kaiser-Meyer-Olkin de adecuación de muestreo } &, 958 \\
\hline \multirow{2}{*}{$\begin{array}{c}\text { Prueba de esfericidad de } \\
\text { Bartlett }\end{array}$} & Aprox. Chi-cuadrado & 8025,639 \\
\cline { 2 - 3 } & gl & 378 \\
\cline { 2 - 3 } & Sig. &, 000 \\
\hline
\end{tabular}

De acuerdo con los resultados presentados en la tabla 3 se obtuvo un resultado de la medida Kaiser-MeyerOlkin de 0,958 muy cercano a 1, lo que indica que es posible realizar el análisis factorial, dado que el test es muy bueno. De acuerdo con la teoría, cuanto más cerca de 1 tenga el valor obtenido del test KMO, implica que la relación entre las variables es alta. Si $\mathrm{KMO} \geq 0.9$, el test es muy bueno; notable para $\mathrm{KMO} \geq 0.8$; mediano para $\mathrm{KMO} \geq 0.7$; bajo para $\mathrm{KMO} \geq 0.6$; y muy bajo para $\mathrm{KMO}<0.5$ (Barbero et al., 2011). Igualmente, en la prueba de esfericidad de Bartlett se obtuvo un nivel de significancia de 0,000 menor que 0,05 lo que valida la aplicación del análisis factorial. Específicamente, la prueba de esfericidad de Bartlett evalúa la aplicabilidad del análisis factorial de las variables analizadas, de acuerdo con la teoría, si la significancia: Sig. ( $p$-valor) $<0.05$ se acepta la $\mathrm{H} 0$ (hipótesis nula) > se puede aplicar el análisis factorial. Posteriormente, se analizaron los resultados de la matriz anti imagen obteniendo valores superiores a 0.9 en todos los ítems, lo anterior, se evidencia en la tabla de comunalidades (Tabla 4) en la cual se establece que todos índices de extracción, obtenidos a través del método de análisis de componentes principales, superan el 0.99. Dado lo anterior, no se hace necesario la eliminación de ningún ítem, ni la realización de nuevos cálculos del análisis factorial. Adicionalmente, el porcentaje de la varianza total explicada, obtenido a través del método de extracción, corresponde a 99,888\%. 
Tabla 4: Análisis factorial: Comunalidades. Método de extracción: análisis de componentes principales.

\begin{tabular}{|c|c|c|}
\hline$N^{\circ}$ Ítem & Inicial & Extracción \\
\hline 1 & 1,000 &, 999 \\
\hline 2 & 1,000 &, 999 \\
\hline 3 & 1,000 &, 999 \\
\hline 4 & 1,000 &, 999 \\
\hline 5 & 1,000 &, 999 \\
\hline 6 & 1,000 & 1,000 \\
\hline 7 & 1,000 &, 999 \\
\hline 8 & 1,000 &, 997 \\
\hline 9 & 1,000 &, 999 \\
\hline 10 & 1,000 &, 997 \\
\hline 11 & 1,000 &, 997 \\
\hline 12 & 1,000 &, 998 \\
\hline 13 & 1,000 &, 999 \\
\hline 14 & 1,000 &, 999 \\
\hline
\end{tabular}

\begin{tabular}{|c|c|c|}
\hline$N^{\circ}$ Ítem & Inicial & Extracción \\
\hline 15 & 1,000 &, 999 \\
\hline 16 & 1,000 &, 999 \\
\hline 17 & 1,000 &, 999 \\
\hline 18 & 1,000 &, 999 \\
\hline 19 & 1,000 &, 999 \\
\hline 20 & 1,000 &, 999 \\
\hline 21 & 1,000 & 1,000 \\
\hline 22 & 1,000 &, 999 \\
\hline 23 & 1,000 &, 997 \\
\hline 24 & 1,000 &, 999 \\
\hline 25 & 1,000 & 1,000 \\
\hline 26 & 1,000 &, 999 \\
\hline 27 & 1,000 &, 999 \\
\hline 28 & 1,000 &, 999 \\
\hline
\end{tabular}

Como resultado de la quinta fase planteada en la metodología, se presentan los pasos para la aplicación del instrumento de recolección de datos propuesto en el presente artículo. Para tal fin, una vez aplicado el instrumento, se sugiere la realización de los siguientes pasos: Paso 1: Se realiza la tabulación de cada uno de los ítems del instrumento de acuerdo con el modelo propuesto en la tabla 5. Cada una de las columnas representa las respuestas de un encuestado, por tanto, en cada celda debe registrarse, en forma numérica, la respuesta seleccionada en cada ítem así: Nunca $=0$, Casi nunca $=1$, A veces $=2$, Casi siempre $=3$, Siempre $=4$.

Tabla 5: Formato para tabulación (paso 1)

\begin{tabular}{|c|l|l|l|l|}
\hline Ítems & Encuestado 1 & Encuestado 2 & Encuestado 3 & Encuestado...n \\
\hline Ítem 1 & & & & \\
\hline Ítem 2 & & & & \\
\hline Ítem 3 & & & & \\
\hline Ítem...n & & & \\
\hline
\end{tabular}

Paso 2: Para el cálculo de la frecuencia (Fa) por ítem, con la función contar.si de Excel, se realiza la tabulación de cada opción de respuesta (Tabla 6). Posteriormente, se estima el porcentaje con referencia a la frecuencia total.

Tabla 6: Formato para el cálculo de la frecuencia por ítem (paso 2).

\begin{tabular}{|c|c|c|c|c|c|c|c|c|c|c|c|c|}
\hline Ítems & \multicolumn{2}{|c|}{ Nunca (0) } & \multicolumn{2}{|c|}{ Casi nunca (1) } & \multicolumn{2}{c|}{ Algunas veces (2) } & Casi siempre (3) & \multicolumn{2}{|c|}{ Siempre (4) } & \multicolumn{2}{c|}{ Total } \\
\hline Ítem 1 & $\mathrm{Fa}$ & $\%$ & $\mathrm{Fa}$ & $\%$ & $\mathrm{Fa}$ & $\%$ & $\mathrm{Fa}$ & $\%$ & $\mathrm{Fa}$ & $\%$ & $\mathrm{Fa}$ & $\%$ \\
\hline Ítem 2 & & & & & & & & & & & & \\
\hline Ítem 3 & & & & & & & & & & & & \\
\hline Ítem...n & & & & & & & & & & & & \\
\hline
\end{tabular}

Paso 3: El paso anterior permite obtener las frecuencias y los porcentajes por ítem, no obstante, debe ser analizado por indicadores. De acuerdo con el cuadro 2 (operacionalización de variable), los siguientes indicadores están integrados por más de un ítem: Experticia, la enseñanza se centra en el alumno, problematizador, capacidad de enseñar en procesos y contextos prácticos/ (ausencia de clase magistral), flexibilidad e innovador. Para el análisis de estos indicadores se requiere promediar las frecuencias de los ítems en cada opción de respuesta. Asimismo, se debe estimar posteriormente, el porcentaje correspondiente.

Paso 4: para el cálculo de la media $(x)$ y la desviación estándar $(s)$ por cada indicador, deben tomarse los datos de los ítems correspondientes en el formato del paso 1 (Tabla 5) y estimar los parámetros con la función promedio (media) y DESVESTA (desviación estándar). Como resultado de los pasos anteriores se obtienen las siguientes tablas para análisis y discusión de resultados. La tabla 7 presenta el ejemplo de la primera subdimensión de instrumento, se debe construir una tabla por cada sub-dimensión presentada en la Tabla 2: Operacionalización de la variable. 
Tabla 7: Resultados de la sub-dimensión conocimiento

\begin{tabular}{|c|c|c|c|c|c|c|c|c|c|c|c|c|c|c|}
\hline \multicolumn{15}{|c|}{ Dimensión: Competencias } \\
\hline \multirow{3}{*}{$\begin{array}{c}\text { Sub- } \\
\text { Dimensión }\end{array}$} & \multirow{3}{*}{$\begin{array}{l}\text { Indicador ( } \\
\text { Ítems) }\end{array}$} & \multicolumn{10}{|c|}{ Escala de Likert } & \multirow{2}{*}{\multicolumn{2}{|c|}{ Total }} & \\
\hline & & \multicolumn{2}{|c|}{$\begin{array}{c}\text { Nunca } \\
(0)\end{array}$} & \multicolumn{2}{|c|}{$\begin{array}{l}\text { Casi nunca } \\
\text { (1) }\end{array}$} & \multicolumn{2}{|c|}{$\begin{array}{c}\text { Algunas veces } \\
\text { (2) }\end{array}$} & \multicolumn{2}{|c|}{$\begin{array}{c}\text { Casi siempre } \\
\text { (3) }\end{array}$} & \multicolumn{2}{|c|}{$\begin{array}{c}\text { Siempre } \\
\text { (4) }\end{array}$} & & & $X \mid s$ \\
\hline & & $\mathrm{Fa}$ & $\%$ & $\mathrm{Fa}$ & $\%$ & $\mathrm{Fa}$ & $\%$ & $\mathrm{Fa}$ & $\%$ & $\mathrm{Fa}$ & $\%$ & $\begin{array}{l}\mathrm{F} \\
\mathrm{a}\end{array}$ & \% & \\
\hline Conocimiento & Experticia $(1,2)$ & & & & & & & & & & & & & \\
\hline
\end{tabular}

Paso 5: para complementar el análisis de los datos, pueden estimarse baremos para el análisis de la media y la desviación estándar. Estos baremos se construyen a partir de los datos máximos y mínimos obtenidos de la aplicación del instrumento (Pertuz et al., 2016). Se presentan algunos ejemplos (Tablas 8 y 9):

Tabla 8: Baremo ponderado para el análisis de las medias.

\begin{tabular}{|c|l|c|l|}
\hline Valor & Alternativa & Intervalos & Categorías \\
\hline 4 & Siempre & $4,20-5,00$ & Muy alta \\
\hline 3 & Casi siempre & $3,40-4,19$ & Alta \\
\hline 2 & Algunas veces & $2,60-3,39$ & Intermedia \\
\hline 1 & Casi nunca & $1,80-2,59$ & Baja \\
\hline 1 & Nunca & $1,00-1,79$ & Ausente \\
\hline
\end{tabular}

Tabla 9: Baremo ponderado para el análisis de la desviación estándar.

\begin{tabular}{|l|l|}
\hline Intervalos & \multicolumn{1}{|c|}{ Categorías } \\
\hline $1,39-1,55$ & Muy alta dispersión (MAD) \\
\hline $1,23-1,38$ & Alta dispersión (AD) \\
\hline $1,07-1,22$ & Dispersión intermedia (DI) \\
\hline $0,91-1,06$ & Baja dispersión (BD) \\
\hline $0,75-0,90$ & Ausente dispersión (AUD) \\
\hline
\end{tabular}

\section{CONCLUSIONES}

El instrumento de recolección de datos presentado en el artículo aporta una herramienta para el desarrollo de programas de formación a formadores en emprendimiento y, consecuentemente, contribuye al desarrollo de actitudes emprendedoras en estudiantes, mediante la práctica docente. El cuestionario propuesto se orienta a identificar las características personales y didáctica utilizada habitualmente en la práctica docente, a través del autoreporte. A partir de la revisión de literatura realizada para construir el instrumento, se concluye que la formación a formadores en emprendimiento es un asunto de actitud, destacándose la empatía, la tolerancia, la flexibilidad, el respeto y la responsabilidad. Asimismo, la practica pedagógica debe centrase en el estudiante, bajo enfoques sistemáticos y sustentables. Específicamente, el instrumento cuenta con 28 ítems, que miden un total de 22 características del docente que forma emprendedores, asociadas a seis componentes de las competencias: conocimiento ( 2 ítems), habilidades (14 ítems), rol social (1 ítem), rasgos de carácter ( 5 ítems), valores ( 3 ítems) y motivos ( 3 ítems). El instrumento fue validado utilizando el coeficiente de Alfa de Cronbach, obteniendo un resultado de 0.88 , adicionalmente, los resultados del análisis factorial validan los ítems incluidos en el cuestionario. La investigación contribuye con el mejoramiento en la formación de emprendedores en la Educación Superior y facilita la formulación de programas de formación a formadores en emprendimiento. El mismo puede ser aplicado a docentes universitarios indistintamente del programa académico. La presente propuesta facilita los procesos de aprendizaje relacionado con el emprendimiento en los estudiantes universitarios.

\section{REFERENCIAS}

Abbasi, B., A. Amatsenin, H. Ashari y A. Gul, Entrepreneurial career adoption model: A way forward, Advanced Science Letters, 23(9), 8023-8030 (2017)

Agudo, J. E., R. Hernández, M. Rico y H. Sánchez, Competencias Transversales: Percepción de su desarrollo en el Grado en Ingeniería en Diseño Industrial y Desarrollo de Productos, Formación Universitaria, 6(5), 39-50 (2013) 
Alles, M., Desarrollo del talento Humano basado en competencias, $3^{a}$ Ed., Ediciones Granica S.A., Buenos Aires, Argentina (2006)

Arunkumar, S., J. Jose Prabhu y otros cinco autores, Entrepreneurial attitude among management students: an empirical study, International Journal of Pure and Applied Mathematics, 119(7), 2389-2399 (2018)

Baeza, M.J., A. Antivilo y L.E. Rehbein, Diseño y Validación de una Escala de Preparatividad Académica para la Educación Superior en Chile, Formación Universitaria, 9(4), 63-74 (2016)

Barbero, M.I., E. Vila y F.P. Holgado, Introducción básica al análisis factorial, Editorial UNED, Madrid, España, Universidad Nacional de Educación a Distancia (2011)

Bello, M.E., Competencias del docente para la formación de capacidad emprendedora en la universidad metropolitana, Departamento de Didáctica, Universidad Metropolitana, Caracas - Venezuela (2008)

Bernal, A. y A. Cárdenas, Assessment of enterprise potential in students. A longitudinal study, Educación XX1, 20 (2), 73$94(2017)$

Caldera, J., F. Rodríguez y J. Moreno, Manual guía para agentes multiplicadores en estímulo y desarrollo de capacidades emprendedoras, CAF, Universidad Metropolitana, INFOEM (Instituto Internacional de Formación Empresarial), Servicio de Atención al Emprendedor (2004)

Cámara de Comercio de Bogotá, El docente como promotor y formador del emprendimiento. Guía básica programa de emprendimiento en la región Bogotá Cundinamarca, Programa de Emprendimiento en la Región Bogotá Cundinamarca, Bogotá D.C. (2009)

Campos, O.R. y G.C. Méndez, La enseñanza del emprendimiento a partir del aprendizaje basado en problemas (abp) en la educación media técnica, Tesis de Magister en Ciencias de la Educación, Facultad de Educación, Maestría en Ciencias de La Educación, Universidad de La Amazonía, Florencia (2012)

Capella, C., J. Gil, M. Martí y P. Ruiz, Construcción de un cuestionario para medir el emprendimiento social en educación física, Pedagogía Social, Revista Interuniversitaria, 28, 169-188 (2016)

Congreso de Colombia. Ley 1014 de 2006. De fomento a la cultura del emprendimiento, Bogotá D.C. (2006)

Daniel, A., R. Costa, M. Pita y C. Costa, Tourism Education: What about entrepreneurial skills? Journal of Hospitality and Tourism Management, 30(1), 65-72 (2017)

David, K., K. Penaluna, E. McCallum y C. Usei, Embedding entrepreneurial skills development in teacher education, Entrepreneurial Learning City Regions: Delivering on the UNESCO 2013, Beijing Declaration on Building Learning Cities Switzerland, Springer, 319-340 (2017)

Del Solar, S., Emprendedores en el aula, Guía para la Formación en Valores y Habilidades Sociales de Docentes y Jóvenes Emprendedores, Fondo Multilateral de Inversiones del Banco Interamericano de Desarrollo, Santiago, Chile (2010)

Domínguez, M., J. Laros y R. Domínguez, Validación de medidas para evaluar habilidades de conducción y evaluación en profesores universitarios, Revista Electrónica de Investigación y Evaluación Educativa, 16(1), 1-12 (2010)

Durand, M.C., Propuesta para promover el espíritu emprendedor a través de la educación, Centro de Competitividad y Estudios Estratégicos de IPAE Acción Empresarial, Perú (2012)

García, J., Evaluation of entrepreneurial personality: Factorial validity of entrepreneurial orientation questionnaire (COE), Revista Latinoamericana de Psicología, 42(1), 41-52 (2010)

Hernandez, S.R., C. Fernandez y P. Baptista, Metodología de la investigación, 4ª Ed., Mc Graw Hill, México D.F. (2014)

Huang, A., C. Morton y J. Libarkin, Entrepreneurship Assessment in Higher Education: A Research Review for Engineering Education Researchers, Journal of Engineering Education, 107(2), 263-290 (2018)

Jaramillo, L., Emprendimiento: Concepto básico en competencias. Universidad del Norte, Instituto de Estudios en Educación, Lumen, 07 (2008)

Manzano, G. y J.C. Ayala, Psychometric properties of Connor-Davidson Resilience Scale in a Spanish sample of entrepreneurs, Psicothema, 25(2), 245-251 (2013)

Ministerio de Comercio, Industria y Turismo, Asociación Colombiana de Universidades, Estado del Arte sobre Emprendimiento Universitario, Bogotá, Colombia (2014)

Mueller, S. y A. Thomas, Culture and entrepreneurial potential: A nine country study of locus of control and innovativeness, Journal of Business Venturing, 16(1), 51-75 (2001)

Munawaroh, The effect of problem-based learning (PBL) method on students critical thinking skils on entrepeneurship practice course (A study on economics education department in the college of education and teachers' training PGRI jombang East Java Indonesia), Journal of Entrepreneurship Education, 21(1) (2018)

Muñiz, J., J. Suárez, I. Pedrosa, E. Fonseca y E. García, Enterprising personality profi le in youth: Components and assessment, Psicothema, 26(4), 545-553 (2014)

Oliver, A. y L. Galiana, Development and Validation of the Escala de Actitudes Emprendedoras para Estudiantes (EAEE), The Spanish Journal of Psychology, 18, E14 (2015) 
Orti, A.M. y J. Clares, Metodologías formativas en los sistemas de capacitación de emprendedores, Universidad de Sevilla, Dialnet. Ayala Calvo, J.C. \& Grupo de Investigación FEDRA, Conocimiento, innovación y emprendedores: camino al futuro (2007)

Papadaki, Š., P. Novák y J. Dvorský, Attitude of university students to entrepreneurship, Economic Annals-XXI, 166(7-8), 100-104 (2017)

Pedrosa, I., J. Suárez, E. García y J. Muñiz, A computerized adaptive test for enterprising personality assessment in youth, Psicothema, 28(4), 471-478 (2016)

Pertuz, V. P., G. Y. Rojas, A. Navarro y L. T. Quintero, Perfil docente y fomento de la cultura del emprendimiento: búsqueda de una relación, Revista Educación y Educadores, 19(1), 29-45 (2016)

Portuguez, M., J.R. Valenzuela y C. Navarro, Diseño y validación de un test de evaluación del potencial en emprendimiento social, Revista de Estudios Cooperativos, 128, 192-211 (2018)

Reynolds, P., N. Bosma y otros cinco autores, Global entrepreneurship monitor: Data collection design and implementation 1998-2003, Small Business Economics, 24(3), 205-231 (2005)

Rodríguez, P., M. Pastor y P. Alonso, University entrepreneurship: how to trigger entrepreneurial intent of undergraduate students, Journal of Science and Technology Policy Management (2018)

Roy, R., F. Akhtar y N. Das, Entrepreneurial intention among science \& technology students in India: extending the theory of planned behavior, International Entrepreneurship and Management Journal, 13, 1013-1041 (2017)

Sepúlveda, J., M. Denegri y otros tres autores, Características emprendedoras personales y alfabetización económica: Una comparación entre estudiantes universitarios del sur de Chile, Interdisciplinaria, 34(1), 107-124 (2017)

Sher, A., S. Adil, y otros tres autores, An investigation of entrepreneurial intentions of agricultural students, Pakistan Journal of Agricultural Sciences, 54(4), 941-945 (2017)

Singh, B., P. Verma y M. Rao, Influence of individual and socio-cultural factors on entrepreneurial intention, Entrepreneurship Education: Experiments with Curriculum, Pedagogy and Target Groups, Springer, Singapore, 149-169 (2017)

Spencer, L. M. y S. M. Spencer, Competence at Work. Models of Superior Performance, John, New York (1993)

Stadler, A. y A. Smith, Entrepreneurship in vocational education: A case study of the Brazilian context, Industry and Higher Education, 31(2), 81-89 (2017)

Tiwari, P., A. Bhat y J. Tikoria, Relationship between entrepreneurship education and entrepreneurial intentions: A validation study, Entrepreneurship Education: Experiments with Curriculum, Pedagogy and Target Groups, Springer, Singapore, 171-188 (2017)

Tognazzo, A., M. Gianecchini y P. Gubitta, Educational context and entrepreneurial intentions of university students: An Italian study, Contemporary Issues in Entrepreneurship Research, Emerald Publishing Limited, 47-74 (2017)

Trivedi, R., Entrepreneurial-intention constraint model: A comparative analysis among post-graduate management students in India, Singapore and Malaysia, Inter. Entrepreneurship and Management Journal, 13, 1239-1261 (2017)

Verzat, C., N. O'Shea y M. Jore, Teaching proactivity in the entrepreneurial classroom, Entrepreneurship and Regional Development, 29(9-10), 975-1013 (2017)

Yi, A. y N. Duval, WhatDrives Engineering Students To Be Entrepreneurs? Evidence ofValidity for an Entrepreneurial Motivation Scale, Journal of Engineering Education, 107(2), 291-317 (2018) 